\title{
Miscibility of Poly(vinyl alcohol)/Poly(methacrylic acid) and Poly(vinyl alcohol/Poly(acrylic acid) Systems II. High-Resolution Solid-State CP/MAS ${ }^{13}$ C NMR Studies
}

\author{
Xiaoqing Zhang, ${ }^{*}$ K. TaKegoshi, and Kunio HiKichI \\ NMR Laboratory and Department of Polymer Science, Faculty of Science, \\ Hokkaido University, Sapporo 060, Japan
}

(Received August 6, 1990)

\begin{abstract}
Miscibility of poly(vinyl alcohol)/poly(methacrylic acid) complexes and poly(vinyl alcohol)/poly(acrylic acid) blends is investigated by high-resolution ${ }^{13} \mathrm{C}$ solid-state NMR method. Observed ${ }^{13} \mathrm{C}$ spectra are discussed in terms of hydrogen-bonding effects on chemical shift. The results indicate that poly(vinyl alcohol) and poly(methacrylic acid) are intimately mixed on a scale of $20-30 \AA$ due to intermolecular hydrogen bonding to form equimolar-ratio complexes. For the poly(vinyl alcohol)/poly(acrylic acid) $=1 / 1$ blend, the two polymers are also miscible, the crystalline phase of PVA is destroyed completely and no detectable domain can be observed for the blend on a scale of $20-30 \AA$. Poly(vinyl alcohol)/poly (acrylic acid) $=2 / 1$ and $1 / 2$ blends are homogenous on a scale of $200-300 \AA$, but heterogeneous on a smaller scale.
\end{abstract}

KEY WORDS Poly(vinyl alcohol) / Poly(methacrylic acid) / Poly(acrylic acid) / High-Resolution ${ }^{13} \mathrm{C}$ Solid-State NMR / Polymer Blends / Polymer Complexes / Miscibility / Hydrogen-Bonding Interaction / Morphology /

For two dissimilar polymers to be miscible, some exothermic intermolecular interactions should occur. Among many possible interactions, the hydrogen-bonding interaction has been studied extensively in recent years. ${ }^{1-6}$ High-resolution ${ }^{13} \mathrm{C}$ solid-state NMR is particularly useful for investigating hydrogenbonding interaction. Helpful information about the hydrogen bonding can be obtained through characteristic downfield shifts of ${ }^{13} \mathrm{C}$ nuclei participating in hydrogen bonding. ${ }^{7,8}$ Although such downfield shift induced by hydrogen bonding in solution reflects an average of bonded and non-bonded states, a rigid structure of hydrogen bonding in the solid state would cause further downfield shift. ${ }^{9,10}$ Furthermore, the high-resolution ${ }^{13} \mathrm{C}$ solidstate NMR method enables us to measure the relaxation time of ${ }^{1} \mathrm{H}$ spins of individual components of polymers through well-resolved ${ }^{13} \mathrm{C}$ signals and to examine the domain structure and miscibility of polymer blends in the solid state. If polymer chains are intimately mixed, fast spin diffusion among ${ }^{1} \mathrm{H}$ spins equalizes spin-lattice relaxation times of all protons. On the other hand, if the domain size of multi-phase structure of polymer blends is larger than the characteristic spin diffusion path length, the relaxation time can be observed separately for each component of polymers. ${ }^{11-18}$

In this work, we examine the hydrogenbonding interaction and miscibility of poly(vinyl alcohol)/poly(methacrylic acid) (PVA/ PMAA) and poly(vinyl alcohol)/poly(acrylic acid) (PVA/PAA) systems. Since PVA, PMAA, and PAA all have strong hydrogen-bonding donors and acceptors, there would be inter-

\footnotetext{
* On leave from Analytical Center, East China Normal University, Shanghai, P. R. China.
} 
molecular hydrogen bonds between two different polymers as well as intramolecular and intermolecular hydrogen bonds among the same kinds of individual polymers. The high-resolution proton NMR study of PVA/ PMAA and PVA/PAA systems in dimethyl sulfoxide (DMSO) solution has been reported in the preceeding paper, ${ }^{19}$ in which the interaction between PVA and PMAA or PAA was observed. It was suggested that the different polymer chains are mixed on a scale less than $4 \AA$. In this paper, we provide some information about intermolecular hydrogen bonding between two different polymers in the solid state by ${ }^{13} \mathrm{C} \mathrm{CP} / \mathrm{MAS}$ NMR techniques. The domain structure and miscibility of PVA/PMAA and PVA/PAA systems on a scales of $20-30 \AA$ to $200-300 \AA$ were also investigated by measuring the ${ }^{1} \mathrm{H}$ spin-lattice relaxation times both in rotating $\left(T_{1 \rho}\right)$ and laboratory frames $\left(T_{1}\right)$.

\section{EXPERIMENTAL}

The molecular weight of PVA is 110,000 , and those of PMAA and PAA are 40,00070,000 and 20,000 -40,000, respectively.

PVA and PAA were mixed in aqueous solution at $\mathrm{pH}=2$ in compositions of $2 / 1,1 / 1$, and $1 / 2$ in monomer-units. The homogenously mixed solutions were obtained. After stirring the mixed solutions for $5 \mathrm{~h}$, the solvent was allowed to evaporate at room temperature for several days and the transparent films were obtained after further drying under reduced pressure at $323 \mathrm{~K}$ for 3 days.

PVA/PMAA samples were prepared by mixing $0.12 \mathrm{wt} \%$ aqueous solutions of PVA and PMAA in monomer-units of $1 / 2$ and $2 / 1$ at $\mathrm{pH}=2$. Prowderish solids appeared as soon as the two solutions were mixed. The mixtures were stirred for one day and then allowed to stand for $20 \mathrm{~h}$ to precipitate the powder solids completely. The solids were filtered, washed and dried under reduced pressure at $323 \mathrm{~K}$ for 3 days. The composition of the resulting samples was found to be $1.1 / 1.0$ for both mixtures, as previous reported. ${ }^{19}$ We denote these samples as PVA/PMAA complexes 1 and 2.

NMR experiments were carried out on a JEOL JNM-GX270 spectrometer operating at resonance frequencies of 270 and $67.8 \mathrm{MHz}$ for ${ }^{1} \mathrm{H}$ and ${ }^{13} \mathrm{C}$, respectively. High-resolution ${ }^{13} \mathrm{C}$ NMR was realized by magic-angle sample sinning (MAS) and high-power spin decoupling. To enhance the signal-to-noise ratio, the crosspolarization (CP) technique was applied. ${ }^{1} \mathrm{H}$ decoupling frequency was chosen to be $3 \mathrm{ppm}$ downfield from tetramethylsilane (TMS). A double-bearing aluminum oxide rotor was used and the setting of the magic angle was monitored by the ${ }^{79} \mathrm{Br}$ NMR spectrum of $\mathrm{KBr}$ incorporated in the rotor. ${ }^{20}$ The contact time for $\mathrm{CP}$ was $1 \mathrm{~ms}$ and the radio-frequency (rf) field strength for both ${ }^{1} \mathrm{H}$ and ${ }^{13} \mathrm{C}$ was about $56 \mathrm{KHz} .{ }^{1} \mathrm{H} T_{1 \rho}$ and $T_{1}$ measurements were made by methods reported elsewhere. ${ }^{12,21}$ The chemical shifts of ${ }^{13} \mathrm{C}$ spectra are reported in ppm relative to TMS by taking the methine carbon of solid adamantane $(29.50 \mathrm{ppm})$ as an external reference standard. Variable-temperature measurements were accomplished using a JEOL MVT temperature controller. Temperature calibration was done by the conventional methanol method. ${ }^{22}$

\section{RESULTS AND DISCUSSION}

The ${ }^{13} \mathrm{C} \mathrm{CP} / \mathrm{MAS}$ spectra of PVA, PMAA, and PVA/PMAA complexes and PVA/PAA blends were measured at various temperatures from 311 to $383 \mathrm{~K}$. No remarkable temperature dependence of the spectra was found in this temperature range. The CP/MAS spectra of PVA, PMAA, and PVA/PMAA complexes at a temperature of $311 \mathrm{~K}$ are shown in Figure 1. The spectra of PVA, PAA, and PVA/PAA blends at $311 \mathrm{~K}$ are shown in Figure 2. Bars in the figures indicate ${ }^{13} \mathrm{C}$ peak positions observed in DMSO- $d_{6}$ solution at a temperature of 

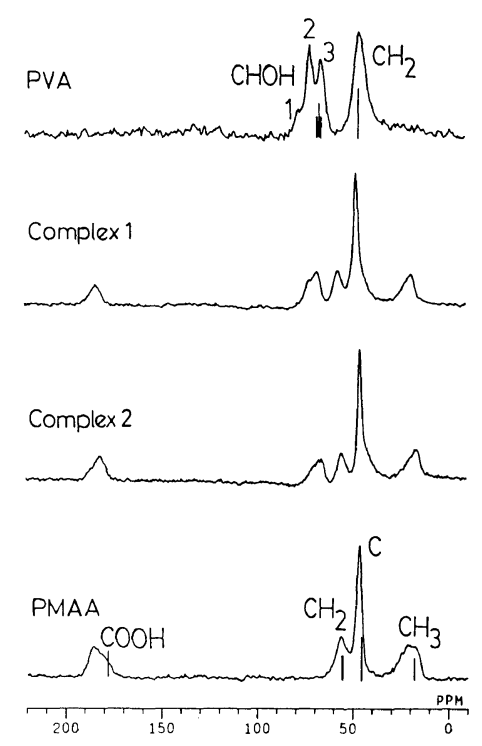

Figure 1. ${ }^{13} \mathrm{C} \mathrm{CP} / \mathrm{MAS}$ spectra of PVA, PMAA and PVA/PMAA complexes at $311 \mathrm{~K}$. Bars indicate peak positions in DMSO- $d_{6}$ solution at $363 \mathrm{~K}$.
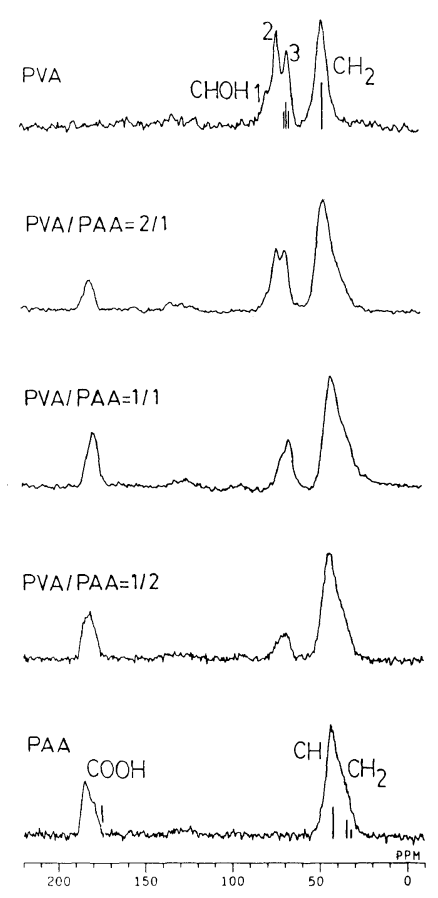

Figure 2. ${ }^{13} \mathrm{C} C P / M A S$ spectra of PVA, PAA and PVA/PAA blends at $311 \mathrm{~K}$. Bars indicate peak position in DMSO- $d_{6}$ solution at $363 \mathrm{~K}$.
$363 \mathrm{~K}$. Peak assignments were based on previous results ${ }^{23-27}$ and shown in the spectra.

Resonances of $\mathrm{COOH}$ carbons of PMAA and PAA and of $\mathrm{CHOH}$ carbon of PVA whose chemical shifts are very sensitive to hydrogen bond formation consist of well-resolved peaks without any overlapping for all samples. For pure PVA, $\mathrm{CHOH}$ carbon shows three peaks in the solid state as previously reported. ${ }^{23}$ In solution, three peaks were also observed, reflecting $m m, m r$, and $r r$ triads. The three $\mathrm{CHOH}$ peaks $(1,2$, and 3$)$ in the solid state appear at lower fields as compared to the triad resonances in solution. Terao et al. interpreted such downfield shifts of $\mathrm{CHOH}$ resonances in the solid state in terms of intramolecular hydrogen bonding. They assigned peak 1 of $\mathrm{CHOH}$ peaks to the $\mathrm{mm}$ triad of $\mathrm{CHOH}$ which is hydrogen bonded to both the two nearest neighbors, peak 2 to $m m$ and $m r$ hydrogen bonded to one of the nearest neighbors, and peak 3 to $m m, m r$, and $r r$ not hydrogen-bonded at all. ${ }^{23}$ Thus, peaks 1 and 2 can be taken as indicators of intramoleclar hydrogen bonding of $\mathrm{OH}$ groups between two cousecutive units of PVA.

For pure PMAA and PAA, line-broadening masks the tacticity splitting of the solid-state spectra. Apart from the broadening, significant downfield shifts of 4-6ppm were found for $\mathrm{COOH}$ carbons in the solid state as compared to those in solution. These downfield shifts would be attributed to hydrogen-bonding enhancement in the rigid solid state.

For PVA/PMAA complexes, the intensities of peaks 1 and 2 of $\mathrm{CHOH}$ carbon resonance decrease significantly. The three-peak characteristic of $\mathrm{CHOH}$ carbon disappears completely, and the whole resonance becomes a single broad peak at about $67.5 \mathrm{ppm}$. The $\mathrm{COOH}$ carbon of PMAA moves to upfield by about 3 ppm relative to that of pure PMAA.

For PVA/PAA blends, a remarkable composition-dependence of the ${ }^{13} \mathrm{C}$ spectra was observed. For PVA/PAA $=2 / 1$ sample, the spectrum still retains the three-peak character- 
istic, although the intensities of peaks 1 and 2 of the $\mathrm{CHOH}$ carbon of PVA decrease noticeably as compared to those of pure PVA. For $\mathrm{PVA} / \mathrm{PAA}=1 / 1$ and $1 / 2$, peaks 1 and 2 become much smaller, and the three-peak characteristic almost disappears. The resonance appears as a single broad line at about $67 \mathrm{ppm}$. Furthermore, upfield shift of the $\mathrm{COOH}$ peak of PAA of $2.7 \mathrm{ppm}$ relative to pure PAA was found for PVA/PAA $=2 / 1$ and $1 / 1$ blends. For PVA/PAA $=1 / 2$, the $\mathrm{COOH}$ peak of PAA appears at $180.3 \mathrm{ppm}$; the shift difference from that of pure PAA is less as compared to those of PVA/PAA $=1 / 1$ and $1 / 2$ blends.

It was reported that if PVA and PMAA are mixed in water solution, white powder is precipitated, and the composition of the precipitates is close to $1 / 1$, irrespective of the initial mixing ratio of PVA and PMAA. Thus, it is suggested that PVA/PMAA forms a complex. The constancy of the composition was confirmed in this work. Therefore, it will be suggested that the interaction undergoing in PVA/PMAA complex is possibly the intermolecular hydrogen bonding between $\mathrm{OH}$ and $\mathrm{COOH}$ groups, as suggested previously. ${ }^{28,29}$ Since peaks 1 and 2 of $\mathrm{CHOH}$ carbon of PVA component are indicators of the intramolecular hydrogen bonding between the two consecutive units, the decrease in intensities of peaks 1 and 2 is probably attributable to the dissociation of the intramolecular hydrogen bonds between the two consecutive units of PVA. The upfield shift of $\mathrm{COOH}$ resonance of PMAA may imply a break of intramolecular hydrogen bonding on complex formation with PVA.

The composition-dependent ${ }^{13} \mathrm{C}$ spectra of PVA/PAA blends can be interpreted in terms of the dissociation of intramolecular hydrogen bonds. When PVA is rich in the system, the equivalent amount of PVA to PAA breaks the intramolecular hydrogen bonding to form miscible blends with PAA and excess PVA still retains intramolecular hydrogen bonding. The observed $\mathrm{CHOH}$ peak reflects the sum of two species, the three-peak characteristic with decreased intensity of peaks 1 and 2 and increased intensity of peak 3 of $\mathrm{CHOH}$ carbon. The upfield shift of $\mathrm{COOH}$ carbon was also observed simultaneously. For PAA rich blend, the situation is just contrary; the dissociation of the intramolecular hydrogen bonds among PVA chains destroys the three-peak characteristic of $\mathrm{CHOH}$ carbon signal while the excess PAA shows little change in $\mathrm{COOH}$ carbon signal.

For polymer blends, it is important to know how homogeneously two different polymers are miscible. The intermolecular hydrogen bonding between two different polymers should cause the systems to be microscopically homogenous. ${ }^{1} \mathrm{H} T_{1 \rho}$ and ${ }^{1} \mathrm{H} T_{1}$ as measured through ${ }^{13} \mathrm{C}$ resonance by $\mathrm{CP} / \mathrm{MAS}$ techniques provide useful information about domain size; typically ${ }^{1} \mathrm{H} T_{1 \rho}$ gives information on a scale of $20-30 \AA$, and $T_{1}$ on a scale of $200-300$ $\AA .{ }^{30-32}$ We measured ${ }^{1} \mathrm{H} T_{1 \rho}$ values of PVA, PMAA, and PAA at three different temperatures and the data are listed in Table I. It was

Table I. ${ }^{1} \mathrm{H} T_{1 \rho}$ values (ms) of PVA, PMAA, and PAA at various temperatures ${ }^{\mathrm{a}}$

\begin{tabular}{|c|c|c|c|c|}
\hline PVA & \multicolumn{2}{|c|}{$\begin{array}{c}\mathrm{CHOH} \\
(76.9,71.2,64.9 \mathrm{ppm})\end{array}$} & \multicolumn{2}{|c|}{$\mathrm{CH}_{2}(43.5 \mathrm{ppm})$} \\
\hline $311 \mathrm{~K}$ & \multicolumn{2}{|c|}{$3.5(43 \%) 9.6(57 \%)$, } & \multicolumn{2}{|c|}{$3.8(46 \%) 9.7(54 \%)$} \\
\hline $339 \mathrm{~K}$ & \multicolumn{2}{|c|}{$2.4(56 \%) 11(44 \%)$} & \multicolumn{2}{|c|}{$2.5(51 \%) 11(49 \%)$} \\
\hline $383 \mathrm{~K}$ & \multicolumn{2}{|c|}{$2.5(54 \%) 10(46 \%)$} & \multicolumn{2}{|c|}{$2.3(53 \%) 11(47 \%)$} \\
\hline PMAA & $\begin{array}{c}\mathrm{COOH} \\
(185.6 \mathrm{ppm})\end{array}$ & $\begin{array}{c}\mathrm{CH}_{2} \\
(55.7 \mathrm{ppm})\end{array}$ & $\begin{array}{c}C \\
(46.2 \mathrm{ppm})\end{array}$ & $\begin{array}{c}\mathrm{CH}_{3} \\
(201 \mathrm{ppm})\end{array}$ \\
\hline $311 \mathrm{~K}$ & 7.0 & 7.2 & 7.6 & 6.7 \\
\hline $339 \mathrm{~K}$ & 7.2 & 7.9 & 7.5 & 7.4 \\
\hline $383 \mathrm{~K}$ & 4.9 & 5.5 & 5.1 & 5.1 \\
\hline PAA & \multicolumn{2}{|c|}{$\mathrm{COOH}(182.2 \mathrm{ppm})$} & \multicolumn{2}{|c|}{$\mathrm{CH}, \mathrm{CH}_{2}(41.5 \mathrm{ppm})$} \\
\hline $331 \mathrm{~K}$ & \multicolumn{2}{|c|}{6.5} & \multicolumn{2}{|c|}{6.9} \\
\hline $339 \mathrm{~K}$ & \multicolumn{2}{|c|}{4.3} & \multicolumn{2}{|c|}{4.2} \\
\hline $383 \mathrm{~K}$ & \multicolumn{2}{|c|}{3.8} & \multicolumn{2}{|c|}{3.9} \\
\hline
\end{tabular}

a PVA shows two components, and values in parenthese represent percentages of two components. 
Table II. ${ }^{1} \mathrm{H} T_{1 \rho}$ values (ms) of PVA/PMAA and PVA/PAA systems at $339 \mathrm{~K}^{\mathrm{a}}$

\begin{tabular}{lcc}
\hline & $\mathrm{COOH}$ & $\mathrm{CHOH}$ \\
\hline PMAA & 7.2 & \\
PVA/PMAA complex 1 & 3.3 & 3.2 \\
PVA/PMAA complex 2 & 3.1 & 3.2 \\
PVA & & $2.4(56 \%), 11(44 \%)$ \\
PVA/PAA $=2 / 1$ & 4.3 & $2.5(49 \%), 10(51 \%)$ \\
PVA/PAA $=1 / 1$ & 2.8 & 2.8 \\
PVA/PAA $=1 / 2$ & 5.3 & $2.4(54 \%), 11(46 \%)$ \\
PAA & 4.3 & \\
\end{tabular}

a PVA and PVA component in PVA/PAA $=2 / 1$ and $1 / 2$ blends show two components, and values in parenthese show percentages of the two components.

found that at $339 \mathrm{~K}$ the value of PVA differs much from those of PMAA and PAA. Therefore, the ${ }^{1} \mathrm{H} T_{1 \rho}$ at $339 \mathrm{~K}$ was measured for PVA/PMAA and PVA/PAA systems (Table II) to study their miscibility.

All carbon resonances of a pure PMAA sample show an identical single-exponential decay with ${ }^{1} \mathrm{H}$ spin-locking time in the $T_{1 \rho}$ experiments, indicating that the fast spin diffusion among protons equalizes the relaxation time of all protons. Similar results were obtained for pure PAA.

On the other hand, all carbon resonances of PVA show non-exponential ${ }^{1} \mathrm{H} T_{1 \rho}$ decay, which can be decomposed into two-exponential components. With increasing temperature, the fast component relaxes more rapidly, while the slow component does not show any remarkable change. Since PVA is a semi-crystalline polymer and the amorphous phase would not be observed by CP techniques because of the weak CP ability ${ }^{23}$ and short proton $T_{1 \rho}$, the slow decay component would be attributed to crystalline phase, and the fast component to an intermediate phase. Because of the different $\mathrm{CP}$ ability between different phases, the ratio of each component does not correspond to the composition of each phase.

For PVA/PMAA complexes, it is worth noting that ${ }^{1} \mathrm{H} T_{1 \rho}$ values for PMAA (observed from the $\mathrm{COOH}$ peak) and for PVA (observed

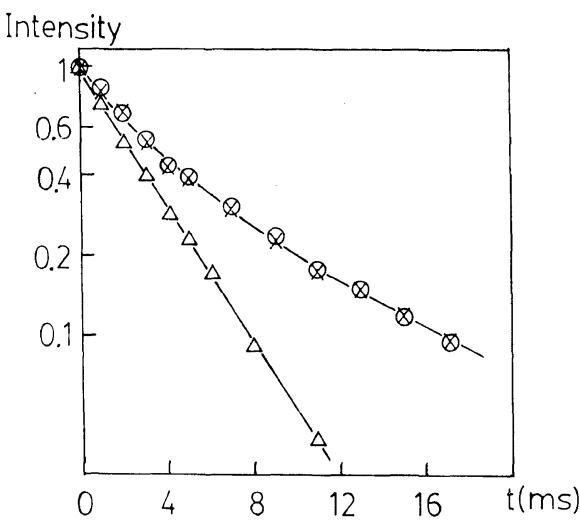

Figure 3. ${ }^{1} \mathrm{H} T_{1 \rho}$ decays of pure PVA $(\times)$ and PVA components (observed from $\mathrm{CHOH}$ peak of PVA) in PVA $/$ PAA $=2 / 1(\bigcirc)$ and PVA/PAA $=1 / 1(\triangle)$ blends, in which the intensity of ${ }^{13} \mathrm{C}$ resonance is plotted against ${ }^{1} \mathrm{H}$ spin-locking time $(t)$.

from the $\mathrm{CHOH}$ peak) are the same at a temperature of $339 \mathrm{~K}$. These results suggest that the spin diffusion among protons of the complex systems is very fast. PVA and PMAA are mixed so intimately that there would be strong dipolar-dipolar interaction between different polymers which cause the strong spin-diffusion coupling interaction between different polymers. Intimate mixing would be caused by the intermolecular hydrogen bonding between PVA and PMAA. No detectable domain can be observed on a scale less than the characteristic spin diffusion path length involved in the $T_{1 \rho}$ measurement. The crystalline phase of PVA also does not exist in PVA/PMAA complexes. The observed $T_{1 \rho}$ values of complexes were found to be the average of the values of pure PMAA and amorphous PVA.

Figure 3 shows the ${ }^{1} \mathrm{H} T_{1 \rho}$ decays of the $\mathrm{CHOH}$ resonances for pure PVA and PVA components in PVA/PAA $=2 / 1$ and $\mathrm{PVA} /$ $\mathrm{PAA}=1 / 1$ bends, in which the intensity of carbon resonance is plotted against the ${ }^{1} \mathrm{H}$ spin-locking time. The ${ }^{1} \mathrm{H} \quad T_{1 \rho}$ decay of PVA $/ \mathrm{PAA}=1 / 1$ blend is also single exponential as similarly as that of PVA/PMAA complexes, suggesting that $\mathrm{PVA} / \mathrm{PAA}=1 / 1$ 
blends form a uniform phase. No detectable domains exist on a scale of $20-30 \AA . T_{1 \rho}$ decays of PVA component of PVA/PAA $=2 / 1$ and $1 / 2$ blends are identical to that of pure PVA and $T_{1 \rho}$ values of PAA observed from the $\mathrm{COOH}$ peak are almost the same as or somewhat larger than those of pure PAA. This implies that on a scale of $20-30 \AA$ the miscibility of both PVA/PAA $=2 / 1$ and $1 / 2$ blends are poor. PVA component in the blends has a similar domain structure as that of pure PVA. In gerenal, the miscibility for crystallineamorphous blends would be better in an amorphous-component rich system than that of crystalline-component rich system, such as poly(vinylidene fluoride)/poly(methyl methacrylate) ${ }^{15}$ poly(phenylene sulphide)/poly(ether sulphone). ${ }^{16}$ poly(vinylidene fluoride)/poly(ethyl methacrylate), ${ }^{33}$ and poly(phenylene sulphide)/polystyrene. ${ }^{34}$ It is of interest to note that for PVA/PAA blends, even in the amorphous component rich system of PVA/$\mathrm{PAA}=1 / 2$, miscibility is still poor.

To explore miscibility on a somewhat larger scale $(200-300 \AA),{ }^{1} \mathrm{H} T_{1}$ of PVA/PAA blends was measured at a temperature of $311 \mathrm{~K}$ and the results are summarized in Table III. Only a single $T_{1}$ value for each sample can be observed, showing that not all domains are detected on this scale. The domain sizes of semi-crystalline PVA and multi-component PVA/PAA blends are all smaller than 200$300 \AA$, and the $T_{1}$ values observed would be the average of $T_{1}$ of all components. On the

Table III. ${ }^{1} \mathrm{H} T_{1}$ values (s) of PVA/PAA blends at $311 \mathrm{~K}$

\begin{tabular}{|c|c|c|c|c|}
\hline & $\mathrm{COOH}$ & $\mathrm{CHOH}$ & $\begin{array}{c}\mathrm{CH}_{2} \text { or } \\
\mathrm{CH}\end{array}$ & Calcd $^{\mathrm{a}}$ \\
\hline PVA & & 7.3 & 7.2 & 7.3 \\
\hline $\mathrm{PVA} / \mathrm{PAA}=2 / 1$ & 5.6 & 5.9 & 5.8 & 5.9 \\
\hline $\mathrm{PVA} / \mathrm{PAA}=1 / 1$ & 5.3 & 5.3 & 5.2 & 5.3 \\
\hline $\mathrm{PVA} / \mathrm{PAA}=1 / 2$ & 3.4 & 3.6 & 3.6 & 4.5 \\
\hline PAA & 3.1 & & 3.0 & 3.1 \\
\hline
\end{tabular}

a Calculated values from eq. 1 (see the text). basis of strong spin-diffusion coupling, the average relaxation time of two-component system can be given as ${ }^{30-32}$

$$
T_{1}^{-1}=\left(N_{1} / N\right) T_{1(1)}^{-1}+\left(N_{2} / N\right) T_{1(2)}^{-1},
$$

where $T_{1(1)}$ and $T_{1(2)}$ denote the observed relaxation times of pure components 1 and 2 with $N_{1}$ and $N_{2}$ protons, respectively, and $N$ is the total number of protons in the system, $N=N_{1}+N_{2}$. The calculated values (shown in Table III as calcd) are in good agreement with the experimental values. This indicates that PVA/PAA blends are completely miscible on a scale of $200-300 \AA$. In gerenal, the physical properties of crystalline-amorphous blends are highly dependent on crystallinity and the properties of the crystalline component in the blends. For PVA/PAA blends, morphology can be controlled by changing the composition of blends. This is beneficial for using high performance polymer blends.

\section{CONCLUSION}

When PVA is mixed with PMAA to form equimolar ratio complexes, the intermolecular hydrogen bonding between PVA and PMAA causes homogenous mixing of the two polymers. No detectable domain can be observed on a scale of $20-30 \AA$ for PVA/PMAA complexes. For $\mathrm{PVA} / \mathrm{PAA}=1 / 1$ blend, the same conclusion can be reached as that for PVA/PMAA complexes. When either PVA or PAA is rich in the blends, the two polymers are miscible on a scale of $200-300 \AA$, but the blends are heterogeneous on a smaller scale.

Acknowledgments. Xiaoqing Zhang is a recipient of the scholarship for foreign students from the Japanese Government. This work was supported by Grants-in-Aid for Scientific Research (Grant No. 02554014 and 02750623) from the Ministry of Education, Science, and Culture of Japan. We are also grateful to $\mathrm{Mr}$. S. Tsuda and Mr. A. Asano for their kind help in NMR experiments. 


\section{REFERENCES}

1. O. Olabisi, L. Robeson, and M. Shaw, "Polymer-Polymer Miscibility," Academic Press, New York, 1979.

2. E. Tsuchida and K. Abe, Adv. Polym. Sci., 45, 000 (1982).

3. D. J. Walsh and S. Rostami, Adv. Polym. Sci., 70, 119 (1985).

4. P. C. Painter, Y. Park, and M. M. Coleman, Macromolecules, 22, 570 (1989); ibid., 22, 580 (1989).

5. M. M. Coleman, D. J. Skrovanek, J. Hu, and P. C. Painter, Macromolecules, 21, 59 (1988).

6. H. Kim, E. M. Pearce, and T. K. Kwei, Macromolecules, 22, 3374 (1984).

7. G. E. Maciel and G. C. Ruben, J. Am. Chem. Soc., 85, 3903 (1963).

8. G. E. Maciel and R. V. James, J. Am. Chem. Soc., 86, 3893 (1964).

9. D. L. VanderHart, W. L. Earl, and A. N. Garroway, J. Magn. Reson., 44, 361 (1981).

10. J. Grobelny, D. M. Rice, F. E. Karasz, and W. J. MacKnight, Macromolecules, 23, 2139 (1990).

11. V. J. McBrierty and D. C. Douglass, J. Polym. Sci. Macromol. Rev., 16, 295 (1981).

12. D. E. Axelson and K. E. Russell, Prog. Polym. Sci., 11, 221 (1985).

13. J. Schaefer, M. D. Sefcik and R. A. Mckay, Macromolecules, 14, 188 (1981).

14. E. O. Stejskal, J. Schaefer, M. O. Sefcik, and R. A. Mckay, Macromolecules, 14, 275 (1981).

15. P. Tekely, F. Laupretre, and L. Monnerie, Polymer, 26, 1081 (1985)

16. X. Zhang and Y. Wang, Polymer, 30, 1867 (1989).

17. C. H. K. Douwel, W. E. J. R. Maas, and W. S.
Veeman, Macromolecules, 23, 406 (1990).

18. X. Zhang, A. Natansohn, and A. Eisenberg, Macromolecules, 23, 412 (1990).

19. X. Zhang, K. Takegoshi, and K. Hikichi, to be published.

20. J. Frye and G. E. Maciel, J. Magn. Reson., 48, 125 (1982).

21. M. J. Suilivan and G. Haciel, Anal. Chem., 54, 1615 (1982).

22. A. L. van Geet, Anal. Chem., 42, 679 (1970).

23. T. Terao, S. Maeda, and A. Saika, Macromolecules, 16, 1536 (1983).

24. J. Schaefer, Macromolecules, 4, 98 (1971).

25. J. D. Cutnell and J. A. Glasel, Macromolecules, 9, 71 (1976).

26. C. Chang, D. D. Muccio, and T. St. Pierre, Macromolecules, 18, 2154 (1985).

27. C. A. Fyfe and M. S. McKinnon, Macromolecules, 19, 1909 (1986).

28. I. S. Okhrimenko and E. B. D'yakonova, Vysokomol. Soedin. Ser. A, 6, 1891 (1964).

29. G. I. Distler, E. B. D'yakonova, I. F. Yefremov, Ye. I. Kortukova, I. S. Okhrimenko, and P. S. Sotnikov, Vysokomol, Soyedin. Ser. A, 8, 1737 (1966).

30. V. J. McBrierty, D. C. Douglass, and T. K. Kwei, Macromolecules, 11, 1265 (1978).

31. B. Albent, R. Jerome, Ph. Teyssie, G. Smyth, N. G. Boyle, and V. J. McBrierty, Macromolecules, 18, 388 (1985).

32. X. Zhang, L. Qiu, D. Wang, and Y. Wang, Chinese J. Polym. Sci., 6(2), 159 (1988).

33. X. Li, Y. Wang, and Y. Yang, Polym. Commun. (China), 5(1), 75 (1987).

34. S. Radhakrishnan and S. Joshi, Eur. Polym. J., 23, 819 (1987). 